Pacific

Journal of

Mathematics

NONLINEAR ALGEBRAIC ANALYSIS OF DELTA SHOCK WAVE SOLUTIONS TO BURGERS' EQUATION

J.-A. MARTI

Volume $210 \quad$ No. 1

May 2003 


\title{
NONLINEAR ALGEBRAIC ANALYSIS OF DELTA SHOCK WAVE SOLUTIONS TO BURGERS' EQUATION
}

\author{
J.-A. MARTI
}

By means of three fundamental structures we can define, in a general way, a sheaf $\mathcal{A}$ of differential algebras containing most of the special cases met in the theory of generalized functions.

A convenient choice of these structures permits us to study Burgers' equation with $\delta$-Dirac measure as initial data, and we can construct a generalized $\delta$-shock wave as an approximate solution, self-similar to the initial data.

\section{Introduction.}

It is not easy to pose and a fortiori to solve the Cauchy problem for Burgers' equation

$$
\frac{\partial u}{\partial t}+\mathfrak{f}(u) \frac{\partial u}{\partial x}=0, \quad t \geq 0
$$

with initial data as irregular as the $\delta$-Dirac measure.

When the data are smooth enough or have weak singularities, the problem has been studied in many classical or generalized ways, but when these singularities become stronger, we first have to describe them.

In this paper, we do not consider solutions of entropy type satisfying the Krushkov (or some other) criteria from the general theory of hyperbolic conservation laws (e.g., [7]).

We try here to study traveling wave solutions self-similar to initial data which can be as singular as $\delta$ or even powers of $\delta$ which do not exist in classical distribution theory. So, we begin by giving a special family $\left(\delta_{\varepsilon}\right)_{\varepsilon}$ of $\delta$-approximations such that for each fixed $\varepsilon>0, p>0$, Burgers' equation has an exact weak self-similar solution corresponding to $\delta_{\varepsilon}^{p}$ as initial data. Then, some models of tsunami or soliton are studied in the same way. However we show that the distribution spaces are not convenient to solve our problem by the help of a limit process.

To get out of this situation we define some other technics of approximation thanks to association processes in $(\mathcal{C}, \mathcal{E}, \mathcal{P})$-algebras (e.g., [11]) which contain most of the special cases met in the literature. Let us give an idea of their construction. 
$\mathbb{K}$ is the real or complex field and $\Lambda$ a set of indices. $\mathcal{C}$ is the factor $\operatorname{ring} A / I$ where $I$ is an ideal of $A$, a given subring of $\mathbb{K}^{\Lambda} .(\mathcal{E}, \mathcal{P})$ is a sheaf of topological $\mathbb{K}$-algebras on a topological space $X$. A sheaf of $(\mathcal{C}, \mathcal{E}, \mathcal{P})$ algebras on $X$ is a sheaf $\mathcal{A}=\mathcal{H} / \mathcal{J}$ of factor algebras where $\mathcal{J}$ is a sheaf of ideals of $\mathcal{H}$, a subsheaf of $\mathcal{E}^{\Lambda}$. The sections of $\mathcal{H}$ (resp. $\mathcal{J}$ ) have to verify some estimations given by means of $\mathcal{P}$ and $A$ (resp. $I$ ). In such algebras we have good tools to pose and solve many non-linear differential problems with irregular data.

The sketch of the procedure is the following: We begin by choosing $\mathcal{E}$ and $\mathcal{P}$ in relationship with the problem. Here, for $\bar{\Omega}=\mathbb{R} \times \mathbb{R}_{+}$, we define $\mathcal{E}(\bar{\Omega})$ as $\mathrm{C}^{\infty}(\bar{\Omega})$ with its usual topology. Then, information about data and equation are taken into account in the construction of $\mathcal{C}$ and finally we can construct a $(\mathcal{C}, \mathcal{E}, \mathcal{P})$-algebra adapted to our problem. It is solved by means of a two-parametric family of special mollifiers with an approximation depending itself upon data and equation peculiarities.

The same methods and technics solve the problem for our models of tsunami or soliton.

\section{The weak form of Burgers' problem.}

The Cauchy problem for Burgers' equation in the following non-conservative form equation:

$$
\frac{\partial u}{\partial t}+\mathfrak{f}(u) \frac{\partial u}{\partial x}=0, t \geq 0, u_{\mid\{t=0\}}=u_{0}
$$

where $\mathfrak{f}(u)=f \circ u$, and $f$ a function of the real variable, has well-known solutions if $f$ and $u_{0}$ are smooth enough and then (1) is equivalent to the conservative form

$$
\frac{\partial u}{\partial t}+\frac{\partial}{\partial x} \mathfrak{F}(u)=0, t \geq 0, u_{\mid\{t=0\}}=u_{0}
$$

when taking $\mathfrak{F}(u)=F \circ u$, with $F^{\prime}=f$.

If $v$ and $\mathfrak{F}(v)$ belong to $L_{\text {loc }}^{1}\left(\mathbb{R} \times \mathbb{R}_{+}\right)$, we define $\tilde{v}$ as

$$
\tilde{v}(x, t)=v(x, t) \text { when } t \geq 0 \text { and } \tilde{v}(x, t)=0 \text { when } t<0
$$

and then it is clear that $\tilde{v}$ belongs to $L_{\text {loc }}^{1}\left(\mathbb{R}^{2}\right) \cap D_{\Gamma}^{\prime}\left(\mathbb{R}^{2}\right)$ and $\mathfrak{F}(\tilde{v})$ belongs to $L_{\text {loc }}^{1}\left(\mathbb{R}^{2}\right) \subset D^{\prime}\left(\mathbb{R}^{2}\right)$ with

$$
D_{\Gamma}^{\prime}\left(\mathbb{R}^{2}\right)=\left\{T \in D^{\prime}\left(\mathbb{R}^{2}\right), \operatorname{supp} T \subset \Gamma=\mathbb{R} \times \mathbb{R}_{+}\right\} .
$$

If $u_{0}$ is in $L_{\text {loc }}^{1}(\mathbb{R})$, to say that $v$ is a weak (distribution) solution of the Cauchy problem (2) means that this problem can be interpreted ([10]) as the equation

$$
\frac{\partial u}{\partial t}+\frac{\partial}{\partial x} \mathfrak{F}(u)=u_{0} \otimes \delta_{t}
$$


to be verified by $\tilde{v}$ in the sense of distibution theory, or explicitly

$$
\begin{aligned}
& -\iint_{t>0}\left(v \frac{\partial}{\partial t} \psi+\mathfrak{F}(v) \frac{\partial}{\partial x} \psi\right)(x, t) d x d t-F(0) \iint_{t<0} \frac{\partial}{\partial x} \psi(x, t) d x d t \\
& =\int u_{0}(x) \psi(0, x) d x, \quad \psi \in C_{0}^{\infty}\left(\mathbb{R}^{2}\right) .
\end{aligned}
$$

Then $u_{0}$ will still be called initial data for (3).

If $u_{0}$ has weak singularities, the problem has been studied in many classical or generalized ways $([\mathbf{1}],[\mathbf{2}],[\mathbf{4}],[\mathbf{8}],[\mathbf{1 8}],[\mathbf{1 9}]$ and many others).

For example, if $\mathfrak{F}(u)=\frac{1}{2} u^{2}$ and $u_{0}=Y$, where $Y$ is the Heaviside function, the search of a self-similar solution, that is to say a solution of the form

$$
u(x, t)=Y(t) u_{0}(x-\varphi(t)), \varphi(0)=0, \varphi^{\prime}>0
$$

leads to the well-known Hugoniot-Renkin condition

$$
\varphi^{\prime}(t)=\frac{1}{2}
$$

But now, if $u_{0}=\delta \in \mathcal{D}^{\prime}(\mathbb{R})$, the research of a solution in the form (4) has no sense for the Cauchy problem (3).

However, if we choose $u_{0}=Y$, and if we search a self-similar solution $u$ of (3) on the form (4) with an unknown strictly increasing function $\varphi \in C^{\infty}(\mathbb{R})$ verifying $\varphi(0)=0$, we can see from

$$
F \circ Y=F(0)+[F(1)-F(0)] Y
$$

for any application $F$ from $\mathbb{R}$ to $\mathbb{R}$, that we have also

$$
\mathfrak{F}(u)=F(0)+[F(1)-F(0)] u .
$$

Then, with classical technics in distribution theory, it is easy to compute

$$
\begin{aligned}
\frac{\partial u}{\partial t} & =-\left(1_{x} \otimes \varphi^{\prime}\right) \delta_{\{x=\varphi(t), t \geq 0\}}+Y_{x} \otimes \delta_{t}, \\
\frac{\partial}{\partial x} \mathfrak{F}(u) & =[F(1)-F(0)] \delta_{\{x=\varphi(t), t \geq 0\}} .
\end{aligned}
$$

This leads to the necessary and sufficient condition for the existence and the uniqueness of the required solution on the form

$$
1_{x} \otimes \varphi^{\prime}=F(1)-F(0)
$$

that is to say

$$
\varphi(t)=[F(1)-F(0)] t .
$$


2.1. Exact weak solutions of Heaviside type. We can try now to search a self-similar solution of $(3)$ in $\mathcal{D}_{\Gamma}^{\prime}\left(\mathbb{R}^{2}\right)$ of the form

$$
u_{h, l}(x, t)=Y(t) u_{0, h, l}(x-\varphi(t))
$$

by taking

$$
u_{0, h, l}(x)=h[Y(x+l)-Y(x-l)]
$$

where $h$ and $l$ are two strictly positive given constants, and then the following holds:

Proposition 1. A necessary and sufficient condition to have an unique solution of (3) in $\mathcal{D}_{\Gamma}^{\prime}\left(\mathbb{R}^{2}\right)$ with the form (6) is

$$
\forall t \geq 0, \varphi(t)=\frac{F(h)-F(0)}{h} t
$$

and then we can explicit the required solution in the form

$u_{h, l}(x, t)=Y(t) h\left[Y\left(x+l-\frac{F(h)-F(0)}{h} t\right)-Y\left(x-l-\frac{F(h)-F(0)}{h} t\right)\right]$.

Proof. We begin to compute

$$
\frac{\partial u_{h, l}}{\partial t}=-h\left(1_{x} \otimes \varphi^{\prime}\right)\left[\delta_{\{x+l=\varphi(t), t \geq 0\}}-\delta_{\{x-l=\varphi(t), t \geq 0\}}\right]+u_{0, h, l} \otimes \delta_{t}
$$

and we have, according to (5)

$$
\mathfrak{F}\left(u_{h, l}\right)=F(0)+[F(h)-F(0)] u_{h, l} .
$$

This gives, in distributional sense

$$
\frac{\partial}{\partial x} \mathfrak{F}\left(u_{h, l}\right)=[F(h)-F(0)]\left[\delta_{\{x+l=\varphi(t), t \geq 0\}}-\delta_{\{x-l=\varphi(t), t \geq 0\}}\right]
$$

and then (6) is a solution of (3) if and only if

$$
h\left(1_{x} \otimes \varphi^{\prime}\right)=F(h)-F(0) .
$$

Hence we deduce the equalities (7) and (8) of the proposition.

Corollary 2. Let

$$
\begin{aligned}
& \delta_{\varepsilon}(x)=u_{\left(0, \frac{1}{2 \varepsilon}, \varepsilon\right)}(x)=\frac{1}{2 \varepsilon}[Y(x+\varepsilon)-Y(x-\varepsilon)], \\
& \tau_{\varepsilon}(x)=u_{\left(0, \frac{1}{2 \varepsilon}, 1\right)}=\frac{1}{2 \varepsilon}[Y(x+1)-Y(x-1)], \\
& \sigma_{\varepsilon}(x)=u_{(0,1, \varepsilon)}=Y(x+\varepsilon)-Y(x-\varepsilon)
\end{aligned}
$$

and $p>0$ a given real number. Then:

(i) Solution (6) of (3) corresponding to $\delta_{\varepsilon}^{p}$ as initial data is given by

$$
u_{p, \varepsilon}(x, t)=Y(t) \frac{1}{(2 \varepsilon)^{p-1}} \delta_{\varepsilon}\left(x-c_{p, \varepsilon} t\right) .
$$


(ii) Solution (6) of (3) corresponding to $\tau_{\varepsilon}^{p}$ as initial data is given by

$$
u_{p, \varepsilon}(x, t)=Y(t) \frac{1}{(2 \varepsilon)^{p-1}} \tau_{\varepsilon}\left(x-c_{p, \varepsilon} t\right) .
$$

(iii) Solution (6) of (3) corresponding to $\sigma_{\varepsilon}^{p}$ as initial data is given by

$$
u_{p, \varepsilon}(x, t)=Y(t) \sigma_{\varepsilon}(x-c t)
$$

with

$$
c_{p, \varepsilon}=(2 \varepsilon)^{p}\left[F\left(\frac{1}{(2 \varepsilon)^{p}}\right)-F(0)\right], c=[F(1)-F(0)] .
$$

Proof. As $Y^{p}=Y$, we still have

$$
\begin{aligned}
\delta_{\varepsilon}^{p}(x) & =\frac{1}{(2 \varepsilon)^{p}}[Y(x+\varepsilon)-Y(x-\varepsilon)]=\frac{1}{(2 \varepsilon)^{p-1}} \delta_{\varepsilon}(x), \\
\tau_{\varepsilon}^{p}(x) & =\frac{1}{(2 \varepsilon)^{p}}[Y(x+1)-Y(x-1)]=\frac{1}{(2 \varepsilon)^{p-1}} \tau_{\varepsilon}(x), \\
\sigma_{\varepsilon}^{p}(x) & =Y(x+\varepsilon)-Y(x-\varepsilon)=\sigma_{\varepsilon}(x)
\end{aligned}
$$

and from (7) and (8), we obtain the results.

\subsection{Approximation in distribution spaces.}

Proposition 3. For some real number $p>0$, we give us $\delta_{\varepsilon}^{p}$ as initial data. Then the generalized sequence of initial data $\left(u_{p, \varepsilon \mid\{t=0\}}\right)_{\varepsilon}$ has no limit in $\mathcal{D}^{\prime}(\mathbb{R})$ except in the case $p=1$ for which we have

$$
\lim _{\substack{\mathcal{D}^{\prime}(\mathbb{R}) \\ \varepsilon \rightarrow 0}}\left(u_{1, \varepsilon \mid\{t=0\}}\right)=\delta .
$$

Moreover:

a) Suppose that we have: $\lim _{x \rightarrow \infty} \frac{F(x)}{x}=L \geq 0$.

If $p<1$, then we have: $\lim _{\mathcal{D}^{\prime}\left(\mathbb{R}^{2}\right)} u_{p, \varepsilon}=0$.

If $p=1$, then we have: $\lim _{\substack{\mathcal{D}^{\prime}\left(\mathbb{R}^{2}\right) \\ \varepsilon \rightarrow 0}} u_{p, \varepsilon}=\delta_{\{x=L t, t \geq 0\}}$.

If $p>1$, then $u_{p, \varepsilon}$ has no limit in $\mathcal{D}^{\prime}\left(\mathbb{R}^{2}\right)$.

b) Suppose that we have: $\lim _{x \rightarrow \infty} \frac{F(x)}{x}=\infty$.

If $p \leq 1$, then we have: $\lim _{\substack{\mathcal{D}^{\prime}\left(\mathbb{R}^{2}\right) \\ \varepsilon \rightarrow 0}} u_{p, \varepsilon}=0$.

If $p>1$, and if: $\lim _{x \rightarrow \infty} \frac{x^{p}}{F\left(x^{p}\right)} \frac{1}{x^{1-p}}=M_{p} \geq 0$, then: $\lim _{\substack{\mathcal{D}^{\prime}\left(\mathbb{R}^{2}\right) \\ \varepsilon \rightarrow 0}} u_{p, \varepsilon}=$ $M_{p} Y_{x} \otimes \delta_{t}$ 


$$
\begin{aligned}
& \quad \text { If } p>1, \text { with: } \lim _{x \rightarrow \infty} \frac{x^{p}}{F\left(x^{p}\right)} \frac{1}{x^{1-p}}=\infty \text {, then } u_{p, \varepsilon} \text { has no limit in } \\
& \mathcal{D}_{\Gamma}^{\prime}\left(\mathbb{R}^{2}\right) \text {. }
\end{aligned}
$$

Proof. The conclusions about the limit in $\mathcal{D}^{\prime}(\mathbb{R})$ of the sequence of initial data are obvious. Now, from Part (i) of Corollary 2 we have, for any test function $\psi \in \mathcal{D}\left(\mathbb{R}^{2}\right)$

$$
\begin{aligned}
\left\langle u_{p, \varepsilon}, \psi\right\rangle & =\frac{1}{(2 \varepsilon)^{p-1}} \iint Y(t) \delta_{\varepsilon}\left(x-c_{p, \varepsilon} t\right) \psi(x, t) d x d t \\
& =\frac{1}{(2 \varepsilon)^{p-1}} \iint Y(t) \delta_{\varepsilon}(u) \psi\left(u+c_{p, \varepsilon} t, t\right) d u d t .
\end{aligned}
$$

From the hypothesis of Part a), we have: $\lim _{\varepsilon \rightarrow 0} c_{p, \varepsilon}=L$, from which we deduce

$$
\begin{aligned}
\lim _{\varepsilon \rightarrow 0} \iint Y(t) \delta_{\varepsilon}(u) \psi\left(u+c_{p, \varepsilon} t, t\right) d u d t & =\int Y(t) \psi(L t, t) d t \\
& =\left\langle\delta_{\{x=L t, t \geq 0\}}, \psi\right\rangle
\end{aligned}
$$

which gives the result of Part a).

So, we can write now

$$
\left\langle u_{p, \varepsilon}, \psi\right\rangle=\frac{1}{c_{p, \varepsilon}} \frac{1}{(2 \varepsilon)^{p-1}} \iint \delta_{\varepsilon}(u) Y(v) \psi\left(u+v, \frac{v}{c_{p, \varepsilon}}\right) d v d u .
$$

From the hypothesis of Part b), we have, for each $p>0$ : $\lim _{\varepsilon \rightarrow 0} c_{p, \varepsilon}=\infty$, from which we deduce

$$
\begin{aligned}
\lim _{\varepsilon \rightarrow 0} \iint \delta_{\varepsilon}(u) Y(v) \psi\left(u+v, \frac{v}{c_{p, \varepsilon}}\right) d v d u & =\int Y(v) \psi(v, 0) d v \\
& =\left\langle\delta_{\{t=0\}}, \psi\right\rangle=\left\langle Y_{x} \otimes \delta_{t}, \psi\right\rangle
\end{aligned}
$$

and we also have

$$
\lim _{\varepsilon \rightarrow 0} \frac{1}{c_{p, \varepsilon}} \frac{1}{(2 \varepsilon)^{p-1}}=\lim _{x \rightarrow \infty} \frac{x^{p}}{F\left(x^{p}\right)} \frac{1}{x^{1-p}}
$$

which gives the result of Part b).

Proposition 4. For some real number $p>0$, we give us $\tau_{\varepsilon}^{p}$ as initial data. Then the generalized sequence of initial data $\left(u_{p, \varepsilon \mid\{t=0\}}\right)_{\varepsilon}$ has no limit in $\mathcal{D}^{\prime}(\mathbb{R})$.

Moreover:

a) Suppose that we have: $\lim _{x \rightarrow \infty} \frac{F(x)}{x}=L \geq 0$, then $u_{p, \varepsilon}$ has no limit in $\mathcal{D}_{\Gamma}^{\prime}\left(\mathbb{R}^{2}\right)$.

b) Suppose that we have: $\lim _{x \rightarrow \infty} \frac{F(x)}{x}=\infty$. 


$$
\begin{aligned}
& \text { If } \lim _{x \rightarrow \infty} \frac{x^{2}}{F(x)}=M \geq 0 \text {, then: } \lim _{\substack{\mathcal{D}^{\prime}\left(\mathbb{R}^{2}\right) \\
\varepsilon \rightarrow 0}} u_{p, \varepsilon}=M(\theta * Y)_{x} \otimes \delta_{t} . \\
& \text { If } \lim _{x \rightarrow \infty} \frac{x^{2}}{F(x)}=\infty \text {, then } u_{p, \varepsilon} \text { has no limit in } \mathcal{D}_{\Gamma}^{\prime}\left(\mathbb{R}^{2}\right) .
\end{aligned}
$$

Proof. We can write

$$
\tau_{\varepsilon}(x)=\frac{1}{2 \varepsilon} \theta(x), \text { with } \theta(x)=Y(x+1)-Y(x-1)
$$

which proves the conclusion about the limit of the sequency of initial data. Then from Part (ii) of Corollary 2 we can compute the corresponding solution as

$$
u_{p, \varepsilon}(x, t)=\frac{1}{(2 \varepsilon)^{p}} Y(t) \theta\left(x-c_{p, \varepsilon} t\right)
$$

for each $(x, t) \in \mathbb{R} \times \mathbb{R}_{+}$. That is to say that, for any test function $\psi \in \mathcal{D}\left(\mathbb{R}^{2}\right)$ we have

$$
\begin{aligned}
\left\langle u_{p, \varepsilon}, \psi\right\rangle & =\frac{1}{(2 \varepsilon)^{p}} \iint Y(t) \theta\left(x-c_{p, \varepsilon} t\right) \psi(x, t) d x d t \\
& =\frac{1}{(2 \varepsilon)^{p}} \iint Y(t) \theta(u) \psi\left(u+c_{p, \varepsilon} t, t\right) d u d t .
\end{aligned}
$$

From the hypothesis of Part a), we have: $\lim _{\varepsilon \rightarrow 0} c_{p, \varepsilon}=L$, from which we deduce

$$
\lim _{\varepsilon \rightarrow 0} \iint Y(t) \theta(u) \psi\left(u+c_{p, \varepsilon} t, t\right) d u d t=\iint Y(t) \theta(u) \psi(u+L t, t) d u d t .
$$

Then $u_{p, \varepsilon}$ has no limit in $\mathcal{D}_{\Gamma}^{\prime}\left(\mathbb{R}^{2}\right)$.

Therefore we also have

$$
\left\langle u_{p, \varepsilon}, \psi\right\rangle=\frac{1}{c_{p, \varepsilon}} \frac{1}{(2 \varepsilon)^{p}} \iint Y(v) \theta(u) \psi\left(u+v, \frac{v}{c_{p, \varepsilon}}\right) d u d v .
$$

From the hypothesis of Part b), we have, for each $p>0$

$$
\lim _{\varepsilon \rightarrow 0} c_{p, \varepsilon}=\infty,
$$

from which we deduce

$$
\begin{aligned}
\lim _{\varepsilon \rightarrow 0} \iint Y(v) \theta(u) \psi\left(u+v, \frac{v}{c_{p, \varepsilon}}\right) d u d v & =\iint Y(v) \theta(u) \psi(u+v, 0) d u d v \\
& =\left\langle(\theta * Y)_{x} \otimes \delta_{t}, \psi\right\rangle
\end{aligned}
$$

and we also have

$$
\lim _{\varepsilon \rightarrow 0} \frac{1}{c_{p, \varepsilon}} \frac{1}{(2 \varepsilon)^{p-1}}=\lim _{x \rightarrow \infty} \frac{x^{2}}{F(x)}
$$

which gives the result of Part $b$ ). 
Proposition 5. For some real number $p>0$, we give us $\sigma_{\varepsilon}^{p}$ as initial data. Then the generalized sequence of initial data $\left(u_{p, \varepsilon \mid\{t=0\}}\right)_{\varepsilon}$ tends to 0 in $\mathcal{D}^{\prime}(\mathbb{R})$. Moreover, the generalized sequence $\left(u_{p, \varepsilon}\right)_{\varepsilon}$ tends to 0 in $\mathcal{D}^{\prime}\left(\mathbb{R}^{2}\right)$.

Proof. We have here

$$
\sigma_{\varepsilon}^{p}(x)=\sigma_{\varepsilon}(x)=Y(x+\varepsilon)-Y(x-\varepsilon)
$$

which proves the conclusion about the limit of the sequency of initial data. From Part (iii) of Corollary 2 we can compute the corresponding solution as

$$
u_{p, \varepsilon}(x, t)=Y(t) \sigma_{\varepsilon}(x-c t)=2 \varepsilon Y(t) \delta_{\varepsilon}(x-c t)
$$

and we have, for any test function $\psi \in \mathcal{D}\left(\mathbb{R}^{2}\right)$

$$
\begin{aligned}
\left\langle u_{p, \varepsilon}, \psi\right\rangle & =2 \varepsilon \iint Y(t) \delta_{\varepsilon}(x-c t) \psi(x, t) d x d t \\
& =2 \varepsilon \iint Y(t) \delta_{\varepsilon}(u) \psi(u+c t, t) d u d t
\end{aligned}
$$

from which we deduce

$$
\lim _{\varepsilon \rightarrow 0} \iint Y(t) \delta_{\varepsilon}(u) \psi(u+c t, t) d u d t=\int Y(t) \psi(c t, t) d t=\left\langle\delta_{\{x=c t, t \geq 0\}}, \psi\right\rangle
$$

which gives the result.

Remark 6. So, the distribution spaces are not convenient to describe the above solutions of Burgers' equation for at least two reasons. First, some of these families of solutions have 0 as limit or no limit in $\mathcal{D}^{\prime}\left(\mathbb{R}^{2}\right)$ and cannot be distinguished, and secondly, except for only two cases, the family of initial data has no limit in $\mathcal{D}^{\prime}(\mathbb{R})$.

\section{The sheaves of $(\mathcal{C}, \mathcal{E}, \mathcal{P})$-algebras.}

In the theory of generalized functions, the following construction extends many points of view met in the literature (e.g., [3], [9], [20]).

We give here a more general definition of the $(\mathcal{C}, \mathcal{E}, \mathcal{P})$-algebra than the first previous one $[\mathbf{1 1}],[\mathbf{1 3}],[\mathbf{1 5}]$. In such algebras we have good tools to pose and solve many non-linear (and even linear) problem with irregular data $[\mathbf{1 2}],[\mathbf{1 4}],[\mathbf{1 6}],[\mathbf{1 7}]$. The topological aspects are studied in [5], [6]. And we will choose the algebraic structure adapted to our Burgers' problem.

\subsection{The algebraic structure.}

a) It is given:

- A set $\Lambda$ of indices,

- a subring $A$ of the ring $\mathbb{K}^{\Lambda},(\mathbb{K}=\mathbb{R}$ or $\mathbb{C})$,

- $A^{+}=\left\{\left(r_{\lambda}\right)_{\lambda} \in A: r_{\lambda} \geq 0\right\}$, 
- the following stability by overestimation property for A: Whenever $\left(\left|s_{\lambda}\right|\right)_{\lambda} \leq\left(r_{\lambda}\right)_{\lambda}$ (that is to say: For each $\lambda$ we have: $\left|s_{\lambda}\right| \leq r_{\lambda}$ ) for any pair $\left(\left(s_{\lambda}\right)_{\lambda},\left(r_{\lambda}\right)_{\lambda}\right) \in \mathbb{K}^{\Lambda} \times A^{+}$, it follows that we have: $\left(s_{\lambda}\right)_{\lambda} \in A$,

- an ideal $I_{A}$ of $A$ with the same stability by overestimation property,

- a sheaf $\mathcal{E}$ of $\mathbb{K}$-algebra on a topological space $X$, such that, for each open set $\Omega$ in $X$, the algebra $\mathcal{E}(\Omega)$ is endowed with the family $\mathcal{P}(\Omega)=\left(p_{i}\right)_{i \in I(\Omega)}$ of semi-norms verifying

$\forall i \in I(\Omega), \exists(j, k, C) \in I(\Omega) \times I(\Omega) \times \mathbb{R}_{+}^{*}: p_{i}(f g) \leq C p_{j}(f) p_{k}(g)$,

- the following property: For two open subsets of $X$ such that $\Omega_{1} \subset$ $\Omega_{2}$, we have $I\left(\Omega_{1}\right) \subset I\left(\Omega_{2}\right)$, and that for every $i \in I\left(\Omega_{1}\right)$ and $u \in \mathcal{E}\left(\Omega_{2}\right)$, we have $p_{i}\left(u \mid{ }_{\Omega_{1}}\right)=p_{i}(u)$.

b) We put

$$
\begin{gathered}
\mathcal{H}_{(A, \mathcal{E}, \mathcal{P})}(\Omega)=\left\{\left(u_{\lambda}\right)_{\lambda} \in[\mathcal{E}(\Omega)]^{\Lambda}, \forall i \in I(\Omega):\left(p_{i}\left(u_{\lambda}\right)\right)_{\lambda} \in A^{+}\right\}, \\
\mathcal{J}_{\left(I_{A}, \mathcal{E}, \mathcal{P}\right)}(\Omega)=\left\{\left(u_{\lambda}\right)_{\lambda} \in[\mathcal{E}(\Omega)]^{\Lambda}, \forall i \in I(\Omega):\left(p_{i}\left(u_{\lambda}\right)\right)_{\lambda} \in I_{A}^{+}\right\}, \\
\mathcal{C}=A / I_{A} .
\end{gathered}
$$

It may be easily seen that $A^{+}$is not a subring of $A$, but is stable under addition and product. It is the same for $I_{A}^{+}$.

At the end, we suppose that the sets $|A|=\left\{\left(\left|r_{\lambda}\right|\right)_{\lambda} \in \mathbb{R}_{+}^{\Lambda}:\left(r_{\lambda}\right)_{\lambda} \in A\right\}$ and $\left|I_{A}\right|=\left\{\left(\left|r_{\lambda}\right|\right)_{\lambda} \in \mathbb{R}_{+}^{\Lambda}:\left(r_{\lambda}\right)_{\lambda} \in I_{A}\right\}$ are respectively subsets of $A$ and $I_{A}$. Then we have: $|A|=A^{+}$and $\left|I_{A}\right|=I_{A}^{+}$.

c) From [11] it follows that under the above hypothesis, we obtain:

\section{Proposition 7.}

(i) $\mathcal{H}_{(A, \mathcal{E}, \mathcal{P})}$ is a sheaf of subalgebras of the sheaf $\mathcal{E}^{\Lambda}$;

(ii) $\mathcal{J}_{\left(I_{A}, \mathcal{E}, \mathcal{P}\right)}$ is a sheaf of ideals of $\mathcal{H}_{(A, \mathcal{E}, \mathcal{P})}$;

(iii) the constant factor sheaf $\mathcal{H}_{(A, \mathbb{K},|.|)} / \mathcal{J}_{\left(I_{A}, \mathbb{K},|.|\right)}$ is exactly the factor ring $\mathcal{C}=A / I_{A}$.

d) Now, we can give the following definition:

Definition 8. We call $(\mathcal{C}, \mathcal{E}, \mathcal{P})$-algebra every factor algebra

$$
\mathcal{A}=\mathcal{H}_{(A, \mathcal{E}, \mathcal{P})} / \mathcal{J}_{\left(I_{A}, \mathcal{E}, \mathcal{P}\right)}
$$

and we denote by $\left[u_{\lambda}\right]$ the class defined by the representative $\left(u_{\lambda}\right)_{\lambda \in \Lambda}$.

By a convenient choice of $\mathcal{C}, \mathcal{E}$ and $\mathcal{P}$ as parameters, we can describe many algebras of generalized functions $[\mathbf{1 1}]$ and define some other operations than algebraic ones such as differentiation, restriction and sheaf embeddings. We also can define local or microlocal analysis. The association or weak equality 
previously defined by Colombeau [3] is a very useful process to study some differential equation in non-conservative form as Burgers' one.

\subsection{Operations, processes and properties.}

3.2.1. Nonlinear functions. If $f$ is a sheaf mapping: $\mathcal{E} \rightarrow \mathcal{E}$, we can define $\tilde{f}=\mathfrak{f}: \mathcal{A} \rightarrow \mathcal{A}$ as a sheaf extension mapping by putting $\mathfrak{f}(u)=\left[f\left(u_{\lambda}\right)\right]$, for any $u \in \mathcal{A}$, defined by the representative $\left(u_{\lambda}\right)_{\lambda \in \Lambda} \in \mathcal{H}_{(A, \mathcal{E}, \mathcal{P})}(\Omega)$. Of course, such an extension needs the following conditions:

If $\left(u_{\lambda}\right)_{\lambda \in \Lambda}$ belongs to $\mathcal{H}_{(A, \mathcal{E}, \mathcal{P})}(\Omega)$, then $\left(f\left(u_{\lambda}\right)\right)_{\lambda}$ belongs to $\mathcal{H}_{(A, \mathcal{E}, \mathcal{P})}$.

If $\left(i_{\lambda}\right)_{\lambda \in \Lambda}$ belongs to $\mathcal{I}_{(A, \mathcal{E}, \mathcal{P})}(\Omega)$, then $\left(f\left(u_{\lambda}+i_{\lambda}\right)-f\left(u_{\lambda}\right)\right)_{\lambda}$ belongs to $\mathcal{I}_{(A, \mathcal{E}, \mathcal{P})}$.

If $P=\sum_{k=0}^{n} f_{k} U^{k}$ is a polynomial with given coefficients in $\mathbb{K}$, or in $\mathcal{E}(X)$, it is easy to see that the above conditions are fulfilled for the sheaf mapping denoted by $\mathcal{P}: \mathcal{E} \rightarrow \mathcal{E}$ and defined by

$$
\mathcal{P}_{\Omega}(e)=\sum_{k=0}^{n} f_{k} e^{k} \text { with } e \in \mathcal{E}(\Omega) .
$$

So, $\mathcal{P}$ has an extension $\widetilde{\mathcal{P}}=\mathfrak{P}: \mathcal{A} \rightarrow \mathcal{A}$ which is the sheaf mapping defined by

$$
\mathfrak{P}_{\Omega}(u)=\left[\sum_{k=0}^{n} f_{k} u_{\lambda}^{k}\right] \text { with } u=\left[u_{\lambda}\right] \in \mathcal{A}(\Omega) .
$$

Remark 9. Therefore we always have polynomials as non-linear functions in any $(\mathcal{C}, \mathcal{E}, \mathcal{P})$-algebra.

3.2.2. Overgenerated rings. In view of applications, it is interesting to define rings generated by some given elements. More precisely, let

$$
B_{p}=\left\{\left(r_{n, \lambda}\right)_{\lambda} \in\left(\mathbb{R}_{+}^{*}\right)^{\Lambda}, n=1,2, \ldots, p\right\}
$$

and $B=\operatorname{span} B_{p}$ be the set of elements of $\left(\mathbb{R}_{+}^{*}\right)^{\Lambda}$ obtained as products, quotients and linear combinations with coefficients in $\mathbb{R}_{+}^{*}$, of elements in $B_{p}$.

Define

$$
A=\left\{\left(a_{\lambda}\right)_{\lambda} \in \mathbb{K}^{\Lambda}, \exists\left(b_{\lambda}\right)_{\lambda} \in B:\left|a_{\lambda}\right| \leq b_{\lambda}\right\} .
$$

It is easy to see that $A$ is a subring of $\mathbb{K}^{\Lambda}$ with the stability by overestimation property. Then, we set the following definition:

Definition 10. $A$ is overgenerated by $B_{p}$. And if $I_{A}$ is some ideal of $A$ with the same stability by overestimation property, we can also say that $\mathcal{C}=A / I_{A}$ is overgenerated by $B_{p}$. 
Example 11. As an ideal $I_{A}$ of $A$, we can take

$$
I_{A}=\left\{\left(a_{\lambda}\right)_{\lambda} \in \mathbb{K}^{\Lambda} \forall\left(b_{\lambda}\right)_{\lambda} \in B:\left|a_{\lambda}\right| \leq b_{\lambda}\right\} .
$$

3.2.3. Relationship with distribution theory. We can adapt the $(\mathcal{C}, \mathcal{E}, \mathcal{P})$-algebras to the multiplication of the distributions in $\mathcal{A}$. So we have first to embed $\mathcal{D}^{\prime}$ in $\mathcal{A}$. If $\left(\varphi_{\varepsilon}\right)_{\varepsilon \in] 0,1]}$ is some given family of mollifiers

$$
\varphi_{\varepsilon}(x)=\frac{1}{\varepsilon^{n}} \varphi\left(\frac{x}{\varepsilon}\right), x \in \mathbb{R}^{n}, \int \varphi(x) d x=1,
$$

we can prove that if one has: $T \in \mathcal{D}^{\prime}\left(\mathbb{R}^{n}\right)$, the convolution product family $\left(T * \varphi_{\varepsilon}\right)_{\varepsilon}$ is a slowly increasing in $\frac{1}{\varepsilon}$ family of smooth functions of $\mathrm{C}^{\infty}\left(\mathbb{R}^{n}\right)$. So we can choose the subring $A$ such that it is overgenerated by some subset $B_{p}$ of $\mathbb{R}_{+}^{[0,1]}$ containing the family $(\varepsilon)_{\varepsilon}$.

Example 12. If $B_{p}$ contains only that family, it is easy to prove that we have

$$
A=\left\{\left(r_{\varepsilon}\right)_{\varepsilon} \in \mathbb{R}^{] 0,1]}, \exists p \in \mathbb{N}, \exists C>0:\left(\left|r_{\varepsilon}\right|\right)_{\varepsilon} \leq\left(\frac{C}{\varepsilon^{p}}\right)_{\varepsilon}\right\},
$$

and we can take

$$
I_{A}=\left\{\left(r_{\varepsilon}\right)_{\varepsilon} \in \mathbb{R}^{00,1]}, \forall q \in \mathbb{N}, \exists D>0:\left(\left|r_{\varepsilon}\right|\right)_{\varepsilon} \leq\left(D \varepsilon^{q}\right)_{\varepsilon}\right\} .
$$

Example 13. Now, for each open set $\Omega$ in $X=\mathbb{R}^{n}$, let us take $\mathcal{E}(\Omega)=$ $\mathrm{C}^{\infty}(\Omega)$ with the usual $\mathcal{P}(\Omega)$ topology of uniform convergency of all the derivatives on the compact subset of $\Omega$. So, we have then:

$$
\begin{array}{r}
\mathcal{H}_{(A, \mathcal{E}, \mathcal{P})}(\Omega)=\left\{\left(u_{\varepsilon}\right)_{\varepsilon} \in\left[C^{\infty}(\Omega)\right]^{00,1]}, \forall K \subset \subset \Omega, \forall \alpha \in \mathbb{N}^{n}, \exists p \in \mathbb{N},\right. \\
\left.\exists C>0:\left(\sup _{x \in K}\left|D^{\alpha} u_{\varepsilon}(x)\right|\right)_{\varepsilon} \leq\left(\frac{C}{\varepsilon^{p}}\right)_{\varepsilon}\right\}, \\
\mathcal{J}_{\left(I_{A}, \mathcal{E}, \mathcal{P}\right)}(\Omega)=\left\{\left(u_{\varepsilon}\right)_{\varepsilon} \in\left[C^{\infty}(\Omega)\right]^{0,1]}, \forall K \subset \subset \Omega, \forall \alpha \in \mathbb{N}^{n}, \forall q \in \mathbb{N},\right. \\
\left.\exists D>0:\left(\sup _{x \in K}\left|D^{\alpha} u_{\varepsilon}(x)\right|\right)_{\varepsilon} \leq\left(D \varepsilon^{q}\right)_{\varepsilon}\right\} .
\end{array}
$$

Then, in this case, the algebra $\mathcal{A}(\Omega)=\mathcal{H}_{(A, \mathcal{E}, \mathcal{P})}(\Omega) / \mathcal{J}_{\left(I_{A}, \mathcal{E}, \mathcal{P}\right)}(\Omega)$ is exactly the Colombeau's simplified one, $[\mathbf{3}]$, and we can embed $\mathcal{D}^{\prime}\left(\mathbb{R}^{n}\right)$ into $\mathcal{A}\left(\mathbb{R}^{n}\right)$ by the mapping

$$
T \rightarrow\left[T * \varphi_{\varepsilon}\right]
$$

because $\left(T * \varphi_{\varepsilon}\right)_{\varepsilon}$ belongs to $\mathcal{H}_{(A, \mathcal{E}, \mathcal{P})}\left(\mathbb{R}^{n}\right)$.

In the same way, with the help of a cutoff function, we can define, for each open set $\Omega$ in $\mathbb{R}^{n}$, an embedding of $\mathcal{D}^{\prime}(\Omega)$ into $\mathcal{A}(\Omega)$, and finally a sheaf embedding: $\mathcal{D}^{\prime} \rightarrow \mathcal{A}$. 
This embedding depends on the choice of the mollifier $\varphi_{\varepsilon}$. And it is easy to define a canonical embedding from $\mathrm{C}^{\infty}$ into $\mathcal{A}$. But we can have a $\mathrm{C}^{k}$ embedding only through the $\mathcal{D}^{\prime}$ one.

3.2.4. On some embeddings. In a more general way, if $E$ is a given sheaf of $\mathbb{K}$-vector spaces with a linear sheaf embedding: $l: \mathcal{E} \rightarrow E$, some problems about existence of embeddings from $\mathcal{E}$ to $\mathcal{A}$ and from $E$ to $\mathcal{A}$ are solved by the following results:

\section{Lemma 14.}

(a) There exists a canonical sheaf morphism $j: \mathcal{E} \rightarrow \mathcal{A}$, i.e., $j_{\Omega}: \mathcal{E}(\Omega) \rightarrow \mathcal{A}(\Omega)$ for each open set $\Omega \subset X$ such that

if and only if we have

$$
\forall f \subset \mathcal{E}(\Omega): j_{\Omega}(f)=\left[(f)_{\lambda}\right]
$$

$$
\begin{aligned}
& (1)_{\lambda} \in A . \\
& \text { (b) There exists a linear sheaf morphism }
\end{aligned}
$$

if and only if, for each $\lambda \in \Lambda$ and each open set $\Omega \subset X$, there exists a linear embedding $k_{\lambda, \Omega}: E(\Omega) \rightarrow \mathcal{E}(\Omega)$, such that we have

$$
\forall u \subset E(\Omega):\left(k_{\lambda, \Omega}(u)\right)_{\lambda} \in \mathcal{H}_{(A, \mathcal{E}, \mathcal{P})}(\Omega) .
$$

Proof. (a) Suppose (18) holds, and suppose $f \neq 0$. There exists $i \in I(\Omega)$ such that $p_{i}(f)=a>0$. Then $\left(p_{i}(f)\right)_{\lambda}=(a)_{\lambda} \in A$. But we have also $\left(\frac{1}{a}\right)_{\lambda} \in A$, and (19) holds. Reciprocally, if (19) holds, we have obviously: $\left(p_{i}(f)\right)_{\lambda} \in A$ for each $f \subset \mathcal{E}(\Omega)$. So, (18) defines a mapping $j_{\Omega}$ from $\mathcal{E}(\Omega)$ to $\mathcal{A}(\Omega)$ which is obviously a morphism of algebras.

(b) Suppose (20) holds. For each $u \in E(\Omega)$, we have $k_{\Omega}(u)=\left[u_{\lambda}\right]$ for some $\left(u_{\lambda}\right)_{\lambda} \in \mathcal{H}_{(A, \mathcal{E}, \mathcal{P})}(\Omega)$. Then, for each $\lambda \in \Lambda$, we can put $k_{\lambda, \Omega}(u)=u_{\lambda}$ and (21) is fulfilled. Conversely, if there exists a linear embedding $k_{\lambda, \Omega}$ : $E(\Omega) \rightarrow \mathcal{E}(\Omega)$, such that $(21)$ holds, when putting $k_{\Omega}(u)=\left[k_{\lambda, \Omega}(u)\right]$, for each $u \in E(\Omega)$, we define a linear sheaf morphism which verifies (20).

Proposition 15. We suppose that the mappings $j$ and $k$ verify the conditions of the previous lemma and moreover there exists a linear sheaf embedding

$$
l: \mathcal{E} \rightarrow E \text { i.e., } l_{\Omega}: \mathcal{E}(\Omega) \rightarrow \mathcal{A}(\Omega) \text { for each open set } \Omega \subset X .
$$

Then the subsheaf $\operatorname{Im} l$ can be canonically equipped with an algebraic structure for which $k$ is an algebra sheaf morphism from $\operatorname{Im} l$ to $\mathcal{A}$ if, for each open set $\Omega \subset X$

$$
\forall u \subset \operatorname{Im} l_{\Omega}:\left(\left(k_{\lambda, \Omega}-l^{-1}\right)(u)\right)_{\lambda} \in \mathcal{I}_{(A, \mathcal{E}, \mathcal{P})}(\Omega) .
$$


Proof. For $u=l^{-1}(f)$ and $v=l^{-1}(g)$ in $\operatorname{Im} l_{\Omega}$, we can define the product $u v$ by putting

$$
u v=l(f g)
$$

which gives $\operatorname{Im} l_{\Omega}$ an algebraic structure.

Now, if $(22)$ is fulfilled, for $u=l(f)$, we have

$$
\forall f \subset \mathcal{E}(\Omega):\left(k_{\lambda, \Omega}(l(f))-f\right)_{\lambda} \in \mathcal{I}_{(A, \mathcal{E}, \mathcal{P})}(\Omega),
$$

which is a necessary and sufficient condition to have

$$
j=k \circ l \text {. }
$$

As $j$ and $l$ are sheaf morphisms of algebras (from $\mathcal{E}$ to $\mathcal{A}$ and $\mathcal{E}$ to $\operatorname{Im} l_{\Omega}$ ), it is the same for $k$ (from $\operatorname{Im} l_{\Omega}$ to $\mathcal{A}$ ).

Remark 16. This result summarizes some questions posed in [20] about the construction of $\mathcal{A}$ from given $\mathcal{E}$ and $E$. For example, let us put $\lambda=$ $(\varphi, \varepsilon) \in \Lambda=\{\varphi\} \times] 0,1]$, with $\mathcal{E}=\mathrm{C}^{\infty}$ and $E=\mathcal{D}^{\prime}$, with a given special mollifier $\varphi \in \mathcal{S}\left(\mathbb{R}^{n}\right)$ such that $\int \varphi(x) x^{\alpha} d x=0$ (resp. 1) for $\alpha \in \mathbb{N}^{n}-\{0\}$ (resp. $\alpha=0$ ). In this case, it is proved in $[\mathbf{3}]$ that for $f=\mathrm{C}^{\infty}(\Omega)$ and $\langle l(f), \psi\rangle=\int f(x) \psi(x) d x$ when $\psi \in \mathcal{D}(\Omega)$, we have

$$
\left(k_{\lambda, \Omega}(l(f))-f\right)_{\lambda}=\left(\varphi_{\varepsilon} *\left(\chi_{\varepsilon} f\right)-f\right)_{\varepsilon} \in \mathcal{I}_{(A, \mathcal{E}, \mathcal{P})}(\Omega)
$$

with $\varphi_{\varepsilon}(x)=\frac{1}{\varepsilon^{n}} \varphi\left(\frac{x}{\varepsilon}\right)$ and $\chi_{\varepsilon} \in \mathcal{D}(\Omega)$ such that $\chi_{\varepsilon}(x)=1$ if $d(x, \partial \Omega)<\varepsilon$ and $d(x, 0)>\frac{1}{\varepsilon}$.

Then, the sheaf embedding $\mathcal{D}^{\prime} \rightarrow \mathcal{A}$ defined, for $T \in \mathcal{D}^{\prime}(\Omega)$ by

$$
k_{\Omega}(T)=\left[\varphi_{\varepsilon} *\left(\chi_{\varepsilon} T\right)\right]
$$

is an algebra sheaf morphism from $\operatorname{Im} l=\mathcal{E}$ to $\mathcal{A}$.

3.2.5. The restriction. When $\mathcal{E}=\mathrm{C}^{\infty}$, the restriction to the submanifold

$$
\left\{x=\left(x_{1}, x_{2}, \ldots, x_{j}, \ldots, x_{n}\right): x_{j}=0\right\} \subset \mathbb{R}^{n}
$$

of the generalized function $u=\left[u_{\lambda}\right] \in \mathcal{A}\left(\mathbb{R}^{n}\right)$ is the generalized one:

\section{Definition 17.}

$$
\left.u\right|_{\left\{x_{j}=0\right\}}=\left[x \rightarrow u_{\lambda}\left(x_{1}, x_{2}, \ldots, x_{j-1, x_{j+1}}, \ldots, x_{n}\right)\right] .
$$

That restriction belongs to a subalgebra of $\mathcal{A}\left(\mathbb{R}^{n}\right)$ which is canonically identified with $\mathcal{A}\left(\mathbb{R}^{n-1}\right)$ and agrees with the similar process met in the literature. 
3.2.6. The derivation. When $\mathcal{E}=\mathrm{C}^{\infty}$, the derivation is defined for each $u=\left[u_{\lambda}\right] \in \mathcal{A}(\Omega)$, by:

$$
\mathcal{D}^{\alpha} u=\left[D^{\alpha} u_{\lambda}\right]
$$

where $D^{\alpha}$ is the classical derivation. Naturally the restriction of $\mathcal{D}^{\alpha}$ to $\mathrm{C}^{\infty}$ or $\mathcal{D}^{\prime}$ agrees with the classical derivation.

Let be $\mathcal{E}$ a sheaf on $X$, with a "derivation operator", that is to say a sheaf endomorphism $D^{\alpha}$ such that:

- $D^{\alpha+\beta}=D^{\alpha} \circ D^{\beta}=D^{\beta} \circ D^{\alpha}$,

- $\forall f, g \in \mathcal{E}(\Omega): D^{\alpha}(f g)=\sum_{\beta \leq \alpha} \frac{\alpha !}{\beta !(\alpha-\beta) !} D^{\beta} f D^{\alpha-\beta} g$.

Then it is possible to define a sheaf endomorphism $\mathcal{D}^{\alpha}$ on $\mathcal{A}$, with the same properties as $D^{\alpha}$ under the only condition:

$$
\text { If }\left(u_{\lambda}\right)_{\lambda} \in J_{\left(I_{A}, \mathcal{E}, \mathcal{P}\right)}(\Omega) \text {, then: }\left(D^{\alpha} u_{\lambda}\right)_{\lambda} \in J_{\left(I_{A}, \mathcal{E}, \mathcal{P}\right)} \text {. }
$$

In this case, we can give as above the following definition:

Definition 18. If $u=\left[u_{\lambda}\right] \in \mathcal{A}(\Omega)$, then, the $\mathcal{D}^{\alpha}$-derivation is given by

$$
\mathcal{D}^{\alpha} u=\left[D^{\alpha} u_{\lambda}\right] \text {. }
$$

3.3. The association process. We suppose that $\Lambda$ is left-filtering for the given (partial) order relation $\prec$.

Let us denote by:

- $\Omega$ every open set in $X$,

- $E$ a given sheaf of topogical $\mathbb{K}$-vector spaces containing $\mathcal{E}$ as a subsheaf,

- $\Phi$ a given application from $\Lambda$ to $\mathbb{K}$ such that $(\Phi(\lambda))_{\lambda}=\left(\Phi_{\lambda}\right)_{\lambda} \in A$.

We also suppose that we have

$$
\mathcal{J}_{\left(I_{A}, \mathcal{E}, \mathcal{P}\right)}(\Omega) \subset\left\{\left(u_{\lambda}\right)_{\lambda} \in \mathcal{H}_{(A, \mathcal{E}, \mathcal{P})}(\Omega): \lim _{E(\Omega)} u_{\lambda}=0\right\} .
$$

Then, for $u=\left[u_{\lambda}\right]$ and $v=\left[v_{\lambda}\right] \in \mathcal{E}(\Omega)$, we define the $\Phi$ - $E$ association.

Definition 19. We denote by

$$
u \underset{E(\Omega)}{\stackrel{\Phi}{\approx}} v
$$

the $\Phi-E$ association between $u$ and $v$ defined by

$$
\lim _{E(\Omega)} \Phi_{\lambda}\left(u_{\lambda}-v_{\lambda}\right)=0 .
$$

That is to say that for each neighbourhood $V$ of 0 for the $E$-topology, there exists $\lambda_{0} \in \Lambda$ such that

$$
\lambda \prec \lambda_{0} \Rightarrow \Phi_{\lambda}\left(u_{\lambda}-v_{\lambda}\right) \in V .
$$


To be sure that the above condition is independant of the representatives of $u$ and $v$, we have to verify that if $\lim _{E(\Omega)} \Phi_{\lambda}\left(w_{\lambda}\right)=0$ holds, for some $\left(w_{\lambda}\right)_{\lambda} \in$

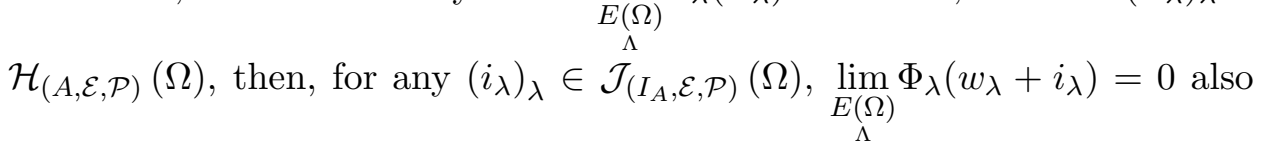
holds. To prove the last condition, it is sufficient to show that

$$
\left(\Phi_{\lambda} i_{\lambda}\right)_{\lambda} \in \mathcal{J}_{\left(I_{A}, \mathcal{E}, \mathcal{P}\right)}(\Omega) .
$$

But for each $i \in I(\Omega)$, we have $p_{i}\left(\Phi_{\lambda} i_{\lambda}\right)=\left|\Phi_{\lambda}\right| p_{i}\left(i_{\lambda}\right)$. And, according to the definitions and the stability properties given in Section 3.1, we have $\left(\left|\Phi_{\lambda}\right|\right)_{\lambda} \in A^{+}$and $\left(p_{i}\left(i_{\lambda}\right)\right)_{\lambda} \in I_{A}^{+}$. Then we also have $\left(\left|\Phi_{\lambda}\right| p_{i}\left(i_{\lambda}\right)\right)_{\lambda} \in I_{A}^{+}$, which proves the required above condition.

Remark 20. When we have $\Phi=1$, it is clear that the above association is weaker than equality. And when taking $\left.\left.E=\mathcal{D}^{\prime}, \mathcal{E}=\mathrm{C}^{\infty}, \Lambda=\right] 0,1\right]$, and $\Phi=1$, we find again the association process defined by Colombeau [3] or Egorov [9] who works in the $(\mathcal{C}, \mathcal{E}, \mathcal{P})$-algebra defined by choosing $\mathcal{C}=\mathbb{C}^{[0,1]} / I_{\mathbb{C}^{0,1]}}$ where $I_{\mathbb{C}^{0,1]}}$ is the ring of complex numbers families $\left(z_{\varepsilon}\right)_{\varepsilon}$ such that $z_{\varepsilon}=0$ if $\varepsilon$ is small enough, and where $\mathcal{E}$ is the sheaf of $C^{\infty}$ complex valued functions. However it is possible to give stronger forms of association, as in the following definition:

Definition 21. Let be $A$ and $I_{A}$ respectively given by (15) and (16).

The $E$-association $u \underset{E(\Omega)}{\approx} v$, between two elements $u$ and $v \in \mathcal{A}(\Omega)$ is defined by: For each $p \in \mathbb{N}$ we have

$$
\lim _{\substack{E(\Omega) \\ \varepsilon \mapsto 0}} \frac{1}{\varepsilon^{p}}\left(u_{\varepsilon}-v_{\varepsilon}\right)=0
$$

Then, by taking $E=\mathcal{E}$, we obtain:

Proposition 22. The $\mathcal{E}$-association is equivalent to the equality in $\mathcal{A}(\Omega)$.

Proof. If one has $u \underset{\mathcal{E}(\Omega)}{\approx} v$, then for each $i \in I(\Omega)$ and $p \in \mathbb{N}^{m}, p_{i}\left(\frac{1}{\varepsilon^{p}}\left(u_{\varepsilon}-v_{\varepsilon}\right)\right)$ is bounded, that is to say: $\left(u_{\varepsilon}-v_{\varepsilon}\right)_{\varepsilon}$ is in the set:

$$
\left.\left.\left\{\left(u_{\varepsilon}\right)_{\varepsilon} \in[\mathcal{E}(\Omega)]^{0,1]}, \forall i \in I(\Omega), \forall \varepsilon \in\right] 0,1\right]:\left(p_{i}\left(u_{\varepsilon}\right)\right)_{e} \in I_{A}^{+}\right\}=\mathcal{J}_{\left(I_{A}, \mathcal{E}, \mathcal{P}\right)}(\Omega) .
$$

\section{The Burgers problem in $(\mathcal{C}, \mathcal{E}, \mathcal{P})$-algebras.}

The first step is to choose the algebra $\mathcal{A}(\mathbb{R})$ and the initial data by means of convenient parameters. The second is to give an as good as possible approximation of corresponding self-similar solutions of the Cauchy problem (1) in a convenient algebra $\mathcal{A}(\bar{\Omega})$ with $\bar{\Omega}=\mathbb{R} \times \mathbb{R}_{+}$. 
4.1. The algebra $\mathcal{A}(\mathbb{R})$ and the initial data. We choose $\mathcal{E}=E=\mathrm{C}^{\infty}$. We define $\mathcal{E}(\mathbb{R})$ as $\mathrm{C}^{\infty}(\mathbb{R})$, with the usual $\mathcal{P}(\mathbb{R})$ topology of uniform convergency of all the derivatives on the compact subset of $\mathbb{R}$ and we define $E(\mathbb{R})$ as $\mathcal{D}^{\prime}(\mathbb{R})$. According to (13), we have here

$$
\mathcal{A}(\mathbb{R})=\mathcal{H}_{(A, \mathcal{E}, \mathcal{P})} / \mathcal{J}_{\left(I_{A}, \mathcal{E}, \mathcal{P}\right)}(\mathbb{R}) .
$$

We choose $\Lambda=] 0,1] \times] 0,1]$, left-filtering for the partial order $\prec$ defined by

$$
\left(\varepsilon_{1}, \eta_{1}\right) \prec\left(\varepsilon_{2}, \eta_{2}\right) \text { if } \varepsilon_{1} \leq \varepsilon_{2} \text { and } \eta_{1} \leq \eta_{2} .
$$

Now, for a given $\varphi \in \mathcal{D}(\mathbb{R})$, with $\operatorname{supp} \varphi=[-1,1], 0 \leq \varphi \leq 1, \varphi(0)=1$, and $\varphi^{(k)}(0)=0$ for each $k \in \mathbb{N}^{*}$, let us consider, for $x \in \mathbb{R}$

$$
\varphi_{(\varepsilon, \eta)}(x)=\frac{1}{2 \varepsilon} \varphi\left(\frac{x}{\eta}\right) .
$$

Then, we define $\delta_{(\varepsilon, \eta)}, \tau_{(\varepsilon, \eta)}, \sigma_{(\varepsilon, \eta)} \in \mathcal{D}(\mathbb{R})$ in the following way:

$$
\begin{gathered}
\delta_{(\varepsilon, \eta)}(x)=\left\{\begin{array}{l}
\left.\varphi_{(\varepsilon, \eta)}(x+\varepsilon)=\frac{1}{2 \varepsilon} \varphi\left(\frac{x+\varepsilon}{\eta}\right) \text { for } x \in\right]-\infty,-\varepsilon[ \\
\frac{1}{2 \varepsilon} \text { for } x \in[-\varepsilon, \varepsilon] \\
\left.\varphi_{(\varepsilon, \eta)}(x-\varepsilon)=\frac{1}{2 \varepsilon} \varphi\left(\frac{x-\varepsilon}{\eta}\right) \text { for } x \in\right] \varepsilon,+\infty[
\end{array}\right. \\
\tau_{(\varepsilon, \eta)}(x)=\left\{\begin{array}{l}
\left.\varphi_{(\varepsilon, \eta)}(x+1)=\frac{1}{2 \varepsilon} \varphi\left(\frac{x+1}{\eta}\right) \text { for } x \in\right]-\infty,-1[ \\
\frac{1}{2 \varepsilon} \text { for } x \in[-1,1] \\
\left.\varphi_{(\varepsilon, \eta)}(x-1)=\frac{1}{2 \varepsilon} \varphi\left(\frac{x-1}{\eta}\right) \text { for } x \in\right] 1,+\infty[
\end{array}\right. \\
\sigma_{(\varepsilon, \eta)}(x)=\left\{\begin{array}{l}
\left.\varphi_{(\varepsilon, \eta)}(x+\varepsilon)=\varphi\left(\frac{x+\varepsilon}{\eta}\right) \text { for } x \in\right]-\infty,-\varepsilon[ \\
1 \text { for } x \in[-\varepsilon, \varepsilon] \\
\left.\varphi_{(\varepsilon, \eta)}(x-\varepsilon)=\varphi\left(\frac{x-\varepsilon}{\eta}\right) \text { for } x \in\right] \varepsilon,+\infty[.
\end{array}\right.
\end{gathered}
$$

It is easy to see that these functions belong to $\mathcal{D}(\mathbb{R})$ and we have: $\operatorname{supp} \delta_{(\varepsilon, \eta)}=\operatorname{supp} \sigma_{(\varepsilon, \eta)}=[-\eta-\varepsilon, \varepsilon+\eta]$ and $\operatorname{supp} \tau_{(\varepsilon, \eta)}=[-\eta-1,1+\eta]$.

Proposition 23. If $A$ is the subring of $\mathbb{R}^{[0,1] \times] 0,1]}$ overgenerated by the set

$$
B_{2}=\left\{(\varepsilon)_{(\varepsilon, \eta)},(\eta)_{(\varepsilon, \eta)}\right\}
$$

then, for any real number $p>0, \Delta^{p}=\left[\delta_{(\varepsilon, \eta)}^{p}\right], \Upsilon^{p}=\left[\tau_{(\varepsilon, \eta)}^{p}\right]$, and $\Xi^{p}=$ $\left[\sigma_{(\varepsilon, \eta)}^{p}\right]$ belong to $\mathcal{A}(\mathbb{R})$. 
Proof. First, we can see that, for each compact $K \Subset \mathbb{R}$ and each $\alpha \in \mathbb{N}$

$$
\sup _{x \in K}\left|\mathcal{D}^{\alpha} \delta_{(\varepsilon, \eta)}^{p}(x)\right| \leq \frac{1}{(2 \varepsilon)^{p}} \frac{1}{\eta^{\alpha}} \sup _{x \in[-1,1]}\left|\mathcal{D}^{\alpha} \varphi^{p}(x)\right|
$$

and then for each seminorm $p_{K, l}$ of the $\mathcal{P}(\mathbb{R})$ topology of $\mathcal{E}(\mathbb{R})=C^{\infty}(\mathbb{R})$, one has: $p_{K, l}\left(\delta_{\varepsilon, \eta}\right) \in A^{+}$, that is to say: $\delta_{\varepsilon, \eta}$ belongs to $\mathcal{H}_{(A, \mathcal{E}, \mathcal{P})}(\mathbb{R})$. The same proof holds for $\tau_{(\varepsilon, \eta)}$ because we have the same estimation for $\sup \left|\mathcal{D}^{\alpha} \tau_{\varepsilon, \eta}^{p}(x)\right|$. Then, from $x \in K$

$$
\sup _{x \in K}\left|\mathcal{D}^{\alpha} \sigma_{(\varepsilon, \eta)}(x)\right| \leq \frac{1}{\eta^{\alpha}} \sup _{x \in[-1,1]}\left|\mathcal{D}^{\alpha} \varphi^{p}(x)\right|
$$

we can easily prove the result for $\Xi$. In this last case, the result holds if $A$ is overgenerated by $\left\{(\eta)_{(\varepsilon, \eta)}\right\}$, and a fortiori by $B_{2}$.

4.2. The algebra $\mathcal{A}(\bar{\Omega})$ and the delta shock wave solutions. For $\bar{\Omega}=\mathbb{R} \times \mathbb{R}_{+}$, we define $\mathcal{E}(\bar{\Omega})$ as $\mathrm{C}^{\infty}(\bar{\Omega})$ with the usual $\mathcal{P}(\bar{\Omega})$ topology of uniform convergency of all the derivatives on the compact subset of $\bar{\Omega}$. And we define $E(\bar{\Omega})$ as $\mathrm{C}^{\infty}(\bar{\Omega})$, with the $\left(p_{\psi}\right)_{\psi \in \mathcal{D}\left(\mathbb{R}^{2}\right)^{\text {-topology, such as }}}$

$$
p_{\psi}(f)=\left|\int_{\Omega} f(x) \psi(x) d x\right|
$$

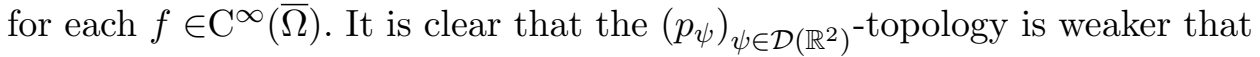
the $\mathcal{P}(\bar{\Omega})$ one.

Then the algebra $\mathcal{A}(\bar{\Omega})$ is defined as

$$
\mathcal{A}(\bar{\Omega})=\mathcal{H}_{(A, \mathcal{E}, \mathcal{P})} / \mathcal{J}_{\left(I_{A}, \mathcal{E}, \mathcal{P}\right)}(\bar{\Omega}) .
$$

The main results are the two following propositions:

Proposition 24. We suppose that $F$ is a polynomial function with real coefficients and increasing with $F(0)=0$, we suppose that $A$ is overgenerated by $B_{2}$, we take $\Phi_{p}(\varepsilon, \eta)=\frac{\varepsilon}{\eta F\left(\frac{1}{(2 \varepsilon)^{p}}\right)}$, and we put respectively $\Delta^{p}$ or $\Upsilon^{p}$ as initial data.Then,

$$
c_{p}=\left[c_{p,(\varepsilon, \eta)} \underset{\mathcal{D}^{\prime}(\mathbb{R})}{\stackrel{\Phi_{p}}{\approx}}\left[(2 \varepsilon)^{p} F\left(\frac{1}{(2 \varepsilon)^{p}}\right)\right]\right.
$$

is a necessary and sufficient condition to have in $\mathcal{A}(\bar{\Omega})$ a $\Phi_{p}$-E approximate solution of the Cauchy problem (1) with the respective self-similar form

$$
\Delta^{p}\left(x-c_{p} t\right)=\left[(x, t) \rightarrow \delta_{(\varepsilon, \eta)}^{p}\left(x-c_{p(\varepsilon, \eta)} t\right)\right]
$$

or

$$
\Upsilon^{p}\left(x-c_{p} t\right)=\left[(x, t) \rightarrow \tau_{(\varepsilon, \eta)}^{p}\left(x-c_{p(\varepsilon, \eta)} t\right)\right]
$$


Proof. To simplify the proof, we suppose that $p=1$. As $F$ is polynomial, $\left(F\left(\frac{1}{2 \varepsilon}\right)\right)_{(\varepsilon, \eta)}$, and $\left(\frac{\varepsilon}{\eta F\left(\frac{1}{2 \varepsilon}\right)}\right)_{(\varepsilon, \eta)}$ belong to $A$. Let be $\mathcal{F}$ the sheaf mapping $\mathcal{E} \rightarrow \mathcal{E}$ induced by $F$, and $\mathfrak{F}: \mathcal{A} \rightarrow \mathcal{A}$ the sheaf mapping extension of $\mathcal{F}$. As $\mathcal{F}\left(\delta_{(\varepsilon, \eta)}\right)$ belongs to $\mathcal{H}_{(A, \mathcal{E}, \mathcal{P})}(\mathbb{R})$, according to the hypothesis on $\mathcal{F}$, we can consider the difference

$$
\mathcal{F}\left(\delta_{(\varepsilon, \eta)}\right)-2 \varepsilon F\left(\frac{1}{2 \varepsilon}\right) \delta_{(\varepsilon, \eta)} .
$$

Then, if $x \in[-\varepsilon, \varepsilon]$, we have

$$
\left[\mathcal{F}\left(\delta_{(\varepsilon, \eta)}\right)\right](x)=2 \varepsilon F\left(\frac{1}{2 \varepsilon}\right) \delta_{(\varepsilon, \eta)}(x) .
$$

For $\psi \in \mathcal{D}(\mathbb{R})$, we compute

$$
\left\langle\left(\mathcal{F}\left(\delta_{(\varepsilon, \eta)}\right)-2 \varepsilon F\left(\frac{1}{2 \varepsilon}\right) \delta_{(\varepsilon, \eta)}\right), \psi\right\rangle=A_{(\varepsilon, \eta)}+B_{(\varepsilon, \eta)}
$$

with

$$
\begin{aligned}
& A_{(\varepsilon, \eta)}=\int_{-\varepsilon-\eta}^{-\varepsilon}\left(F\left[\delta_{(\varepsilon, \eta)}(x)\right]-F\left(\frac{1}{2 \varepsilon}\right) \varphi\left(\frac{x+\varepsilon}{\eta}\right)\right) \psi(x) d x, \\
& B_{(\varepsilon, \eta)}=\int_{\varepsilon}^{\varepsilon+\eta}\left(F\left[\delta_{(\varepsilon, \eta)}(x)\right]-F\left(\frac{1}{2 \varepsilon}\right) \varphi\left(\frac{x-\varepsilon}{\eta}\right)\right) \psi(x) d x .
\end{aligned}
$$

As $F$ is increasing, we have when $x \in[-\varepsilon-\eta,-\varepsilon]$

$$
\left|F\left(\frac{1}{2 \varepsilon}\right) \varphi\left(\frac{x+\varepsilon}{\eta}\right)-F\left[\delta_{(\varepsilon, \eta)}(x)\right]\right| \leq F\left(\frac{1}{2 \varepsilon}\right)
$$

and then

$$
\left|A_{(\varepsilon, \eta)}\right| \leq F\left(\frac{1}{2 \varepsilon}\right) \int_{-\varepsilon-\eta}^{-\varepsilon}|\psi(x)| d x .
$$

Therefore, we have finally

$$
\left|A_{(\varepsilon, \eta)}\right| \leq C \eta F\left(\frac{1}{2 \varepsilon}\right)
$$

for some positive constant $C$, with a similar estimation for $B_{(\varepsilon, \eta)}$. Then we have

$$
\lim _{\substack{\mathcal{D}^{\prime}(\mathbb{R}) \\(\varepsilon, \eta) \rightarrow 0}} \frac{\varepsilon}{\eta F\left(\frac{1}{2 \varepsilon}\right)}\left(\mathcal{F}\left(\delta_{(\varepsilon, \eta)}\right)-2 \varepsilon F\left(\frac{1}{2 \varepsilon}\right) \delta_{(\varepsilon, \eta)}\right)=0 .
$$

From the hypothesis on $\mathcal{F}$ and $A$ we can see that $\left(\mathcal{F}\left(\delta_{(\varepsilon, \eta)}\right)\right)_{(\varepsilon, \eta)}$ and $\left(2 \varepsilon F\left(\frac{1}{2 \varepsilon}\right) \delta_{(\varepsilon, \eta)}\right)_{(\varepsilon, \eta)}$ belong to $\mathcal{H}_{(A, \mathcal{E}, \mathcal{P})}$ and $\left(\frac{\varepsilon}{\eta F\left(\frac{1}{2 \varepsilon}\right)}\right)_{(\varepsilon, \eta)}$ belongs to $A$. 
That is to say

$$
\left[\mathcal{F}\left(\delta_{(\varepsilon, \eta)}\right)\right] \underset{\mathcal{D}^{\prime}(\mathbb{R})}{\stackrel{\Phi}{\approx}}\left[2 \varepsilon F\left(\frac{1}{2 \varepsilon}\right) \delta_{(\varepsilon, \eta)}\right]
$$

according to our definition of association process, with $\Phi_{1}(\varepsilon, \eta)=\frac{\varepsilon}{\eta F\left(\frac{1}{2 \varepsilon}\right)}$.

And that is a good approximation because if $p$ is given as large as we want, we can have

$$
\Phi_{1}(\varepsilon, \eta)>\frac{1}{\varepsilon^{p}} \text { by choosing } \eta<\frac{\varepsilon^{p+1}}{F\left(\frac{1}{2 \varepsilon}\right)} .
$$

Now, if we put $\Delta=\left[\delta_{(\varepsilon, \eta)}\right] \in \mathcal{A}(\mathbb{R})$, we can write

$$
\mathfrak{F}(\Delta) \underset{\mathcal{D}^{\prime}(\mathbb{R})}{\stackrel{\Phi_{1}}{\approx}}\left[2 \varepsilon F\left(\frac{1}{2 \varepsilon}\right)\right] \Delta \text {. }
$$

Let be $\mathfrak{f}: \mathcal{A} \rightarrow \mathcal{A}$ the sheaf mapping defined from $\mathcal{F}: \mathcal{E} \rightarrow \mathcal{E}$ and $F$ by the derivation $F^{\prime}=f$. It is easy to see that if one has: $\mathfrak{F}(u) \underset{\mathcal{D}^{\prime}(\mathbb{R})}{\stackrel{\Phi_{1}}{\approx}} v$, with $u$ and $v$ in $\mathcal{A}(\mathbb{R})$, then we have: $\mathfrak{f}(u) u^{\prime} \underset{\mathcal{D}^{\prime}(\mathbb{R})}{\stackrel{\Phi}{\approx}} v^{\prime}$ in the sense of generalized functions. And then we have also

$$
\mathfrak{f}(\Delta) \Delta^{\prime} \underset{\mathcal{D}^{\prime}(\mathbb{R})}{\stackrel{\Phi_{1}}{\approx}}\left[2 \varepsilon F\left(\frac{1}{2 \varepsilon}\right)\right] \Delta^{\prime}
$$

Now, for $(x, t) \in \bar{\Omega}$, for each compact $K \Subset \mathbb{R}$ and each $\left(k_{1}, k_{2}\right) \in \mathbb{N} \times \mathbb{N}$ we have $\sup _{x \in K}\left|\mathcal{D}^{\left(k_{1}, k_{2}\right)} \delta_{(\varepsilon, \eta)}\left(x-c_{1,(\varepsilon, \eta)} t\right)\right| \leq \frac{1}{2 \varepsilon} \frac{1}{\eta^{k_{1}+k_{2}}}\left|c_{1,(\varepsilon, \eta)}\right|^{k_{2}} \sup _{x \in[-1,1]}\left|\mathcal{D}^{k_{1}+k_{2}} \varphi(x)\right|$ from which it is easy to prove that the family $\left((x, t) \rightarrow \delta_{(\varepsilon, \eta)}\left(x-c_{1(\varepsilon, \eta)} t\right)\right)_{(\varepsilon, \eta)}$ belongs to $\mathcal{H}_{(A, \mathcal{E}, \mathcal{P})}(\bar{\Omega})$. Then, with $c_{1}=\left[c_{1(\varepsilon, \eta)}\right]$, we can put

$$
\Delta\left(x-c_{1} t\right)=\left[(x, t) \rightarrow \delta_{(\varepsilon, \eta)}\left(x-c_{1,(\varepsilon, \eta)} t\right)\right]
$$

and prove, from (32) that we have

$$
\mathfrak{F}\left(\Delta\left(x-c_{1} t\right)\right) \underset{E(\bar{\Omega})}{\stackrel{\Phi_{1}}{\approx}}\left[2 \varepsilon F\left(\frac{1}{2 \varepsilon}\right)\right] \Delta\left(x-c_{1} t\right) .
$$

To do that, we first have to compute

$$
D_{(\varepsilon, \eta)}=p_{\psi}\left(\Phi_{1}(\varepsilon, \eta) G_{(\varepsilon, \eta)}\right)
$$

with

$$
G_{(\varepsilon, \eta)}(x, t)=\left(F\left(\delta_{(\varepsilon, \eta)}\left(x-c_{1(\varepsilon, \eta)} t\right)\right)-2 \varepsilon F\left(\frac{1}{2 \varepsilon}\right) \delta_{(\varepsilon, \eta)}\left(x-c_{1,(\varepsilon, \eta)} t\right)\right) .
$$


Thus we can write

$$
D_{(\varepsilon, \eta)}=\left|\iint Y(t) \Phi_{1}(\varepsilon, \eta) G_{(\varepsilon, \eta)}(x, t) \psi(x, t) d x d t\right|=\int H_{(\varepsilon, \eta)}(t) d t
$$

where

$$
\begin{aligned}
H_{(\varepsilon, \eta)}(t)=\int & Y(t) \Phi_{1}(\varepsilon, \eta)\left[F\left(\delta_{(\varepsilon, \eta)}(u)\right)\right. \\
& \left.-2 \varepsilon F\left(\frac{1}{2 \varepsilon}\right) \delta_{(\varepsilon, \eta)}(u)\right] \psi\left(u+c_{1(\varepsilon, \eta)} t, t\right) d u .
\end{aligned}
$$

From (31), we can see that for each $t \in \mathbb{R}: \lim _{(\varepsilon, \eta) \rightarrow 0} H_{\varepsilon, \eta}(t)=0$. Moreover, if $A>0$ is chosen such that $\operatorname{supp} \psi \subset[-A, A] \times[-A, A]$, we can see from (34) that we have

$$
\left|H_{(\varepsilon, \eta)}(t)\right| \leq C \chi_{[0, A]}
$$

for some constant $C, \chi_{[0, A]}$ being the characteristic function of $[0, A]$, and so, by the Lebesgue majorant convergence theorem, we obtain: $\lim _{(\varepsilon, \eta) \rightarrow 0} D_{(\varepsilon, \eta)}=$ 0 , which proves $(33)$.

Now we can compute

$$
\begin{aligned}
\frac{\partial}{\partial t} \Delta\left(x-c_{1} t\right) & =\left[(x, t) \rightarrow-c_{1,(\varepsilon, \eta)} \delta_{(\varepsilon, \eta)}^{\prime}\left(x-c_{1,(\varepsilon, \eta)} t\right)\right] \\
& =-\left[c_{1,(\varepsilon, \eta)}\right] \Delta^{\prime}\left(x-c_{1} t\right)
\end{aligned}
$$

and from (33)

$$
\frac{\partial}{\partial x} \mathfrak{F}(\Delta(x-c t))=\mathfrak{f}\left(\Delta\left(x-c_{1} t\right)\right) \Delta^{\prime}\left(x-c_{1} t\right) \underset{E(\bar{\Omega})}{\stackrel{\Phi_{1}}{\approx}}\left[2 \varepsilon F\left(\frac{1}{2 \varepsilon}\right)\right] \Delta^{\prime}\left(x-c_{1} t\right) .
$$

So, we have proved that the condition is necessary. And it is obviously sufficient. Particularly, we can take $c_{1,(\varepsilon, \eta)}=2 \varepsilon F\left(\frac{1}{2 \varepsilon}\right)$.

Instead of $\mathcal{F}\left(\delta_{(\varepsilon, \eta)}\right)-2 \varepsilon F\left(\frac{1}{2 \varepsilon}\right) \delta_{(\varepsilon, \eta)}=0$ on $[-\varepsilon, \varepsilon]$ we have: $\mathcal{F}\left(\tau_{(\varepsilon, \eta)}\right)-$ $F\left(\frac{1}{2 \varepsilon}\right) \tau_{(\varepsilon, \eta)}=0$ on $[-1,1]$. But on $[-\eta-1,-1], \mathcal{F}\left(\tau_{(\varepsilon, \eta)}\right)-F\left(\frac{1}{2 \varepsilon}\right) \tau_{(\varepsilon, \eta)}$ is deduced by translation from $\mathcal{F}\left(\delta_{(\varepsilon, \eta)}\right)-2 \varepsilon F\left(\frac{1}{2 \varepsilon}\right) \delta_{(\varepsilon, \eta)}$. So, the same estimations, computations and conclusions holds for $\Upsilon\left(x-c_{1} t\right)$ as well as for $\Delta\left(x-c_{1} t\right)$.

The case $p>0$ follows with slight modifications.

Proposition 25. We suppose that $F$ is a polynomial function with real coefficients and increasing with $F(0)=0$, we suppose that $A$ is overgenerated by

$$
B_{2}=\left\{(\varepsilon)_{(\varepsilon, \eta)},(\eta)_{(\varepsilon, \eta)}\right\}
$$


we take $\Psi(\varepsilon, \eta)=\frac{\varepsilon}{\eta F(1)}$, and for any integer $p \geq 1$, we put $\Sigma^{p}$ as initial data. Then,

$$
d=\left[d_{(\varepsilon, \eta)}\right] \underset{] 0,1] \times] 0,1]}{\stackrel{\Psi}{\approx}}[F(1)]
$$

is a necessary and sufficient condition to have in $\mathcal{A}(\bar{\Omega})$ a $\Psi-E$ approximate solution of the Cauchy problem (1) with the self-similar form

$$
\Sigma^{p}(x-d t)=\left[(x, t) \rightarrow \sigma_{(\varepsilon, \eta)}^{p}\left(x-d_{(\varepsilon, \eta)} t\right)\right] .
$$

Proof. We can consider the difference

$$
\mathcal{F}\left(\sigma_{(\varepsilon, \eta)}^{p}\right)-F(1) \sigma_{(\varepsilon, \eta)}^{p} .
$$

Then if $x \in[-\varepsilon, \varepsilon]$, we have

$$
\left[\mathcal{F}\left(\sigma_{(\varepsilon, \eta)}^{p}\right)\right](x)=F(1) \sigma_{(\varepsilon, \eta)}^{p}(x) .
$$

For $\psi \in \mathcal{D}(\mathbb{R})$, we compute

$$
\begin{aligned}
\langle & \left.\left(\mathcal{F}\left(\sigma_{(\varepsilon, \eta)}^{p}\right)-F(1) \sigma_{(\varepsilon, \eta)}^{p}\right), \psi\right\rangle \\
= & \int_{-\varepsilon-\eta}^{-\varepsilon}\left(\mathcal{F}\left(\sigma_{(\varepsilon, \eta)}^{p}\right)-F(1) \sigma_{(\varepsilon, \eta)}^{p}\right)(x) \psi(x) d x \\
& +\int_{\varepsilon}^{\varepsilon+\eta}\left(\mathcal{F}\left(\sigma_{(\varepsilon, \eta)}^{p}\right)-F(1) \sigma_{(\varepsilon, \eta)}^{p}\right)(x) \psi(x) d x \\
= & A_{(\varepsilon, \eta)}^{\prime}+B_{(\varepsilon, \eta)}^{\prime} .
\end{aligned}
$$

We can compute

$$
A_{(\varepsilon, \eta)}^{\prime}=\int_{-\varepsilon-\eta}^{-\varepsilon}\left(F\left[\sigma_{(\varepsilon, \eta)}^{p}(x)\right]-F(1) \varphi^{p}\left(\frac{x+\varepsilon}{\eta}\right)\right) \psi(x) d x .
$$

As $F$ is increasing, we have when $x \in[-\varepsilon-\eta,-\varepsilon]$

$$
\left|F(1) \varphi^{p}\left(\frac{x+\varepsilon}{\eta}\right)-F\left[\sigma_{(\varepsilon, \eta)}^{p}(x)\right]\right| \leq F(1)
$$

and then

$$
\left|A_{(\varepsilon, \eta)}^{\prime}\right| \leq F(1) \int_{-\varepsilon-\eta}^{-\varepsilon}|\psi(x)| d x .
$$

Therefore, we have finally

$$
\left|A_{(\varepsilon, \eta)}^{\prime}\right| \leq C \eta F(1)
$$

for some positive constant $C$, with a similar estimation for $B_{(\varepsilon, \eta)}^{\prime}$. Then we have 


$$
\lim _{\substack{\mathcal{D}^{\prime}(\mathbb{R}) \\(\varepsilon, \eta) \rightarrow 0}} \frac{\varepsilon}{\eta F(1)}\left(\mathcal{F}\left(\sigma_{(\varepsilon, \eta)}^{p}\right)-F(1) \sigma_{(\varepsilon, \eta)}^{p}\right)=0 .
$$

From the hypothesis on $\mathcal{F}$ and $A$ it follows that $\left(\mathcal{F}\left(\sigma_{(\varepsilon, \eta)}^{p}\right)\right)_{(\varepsilon, \eta)}$ and $\left(F(1) \sigma_{(\varepsilon, \eta)}^{p}\right)_{(\varepsilon, \eta)}$ belong to $\mathcal{H}_{(A, \mathcal{E}, \mathcal{P})}(\mathbb{R})$, with $\left(\frac{\varepsilon}{\eta F(1)}\right)_{(\varepsilon, \eta)}$ in $A$. That is to say

$$
\left[\mathcal{F}\left(\sigma_{(\varepsilon, \eta)}^{p}\right)\right] \underset{\mathcal{D}^{\prime}(\mathbb{R})}{\stackrel{\Psi}{\approx}}\left[F(1) \sigma_{(\varepsilon, \eta)}^{p}\right] .
$$

The next steps can be easily deduce from the previous proof, with slight modifications, and we can choose $F(1)$ as the speed of propagation of the $\Psi$-approximate solution.

Conclusion 26. For any $p>0$, one can say that $\Delta^{p}\left(x-c_{p} t\right), \Upsilon^{p}\left(x-c_{p} t\right)$, $\Sigma^{p}(x-d t)$ are some modelizations of $p$-power of delta-waves, tsunamis, or solitons and approximate self-similar solutions of Burgers' equation. The generalized function $\Sigma^{p}(x-d t)$ propagates approximatively with a finite speed $d=F(1)$, but $\Delta^{p}\left(x-c_{p} t\right)$ and $\Upsilon^{p}\left(x-c_{p} t\right)$ have the same generalized speed $c_{p} \approx\left[(2 \varepsilon)^{p} F\left(\frac{1}{(2 \varepsilon)^{p}}\right)\right]$ depending only upon the generalized height (and not thickness) of the initial data $\Delta^{p}$ and $\Upsilon^{p}$. And in each case, the solution lies in an algebra adapted to the problem by the choice of the algebra $\mathcal{E}(\bar{\Omega})$ and the ring $\mathcal{C}$ of generalized numbers. Indeed $\mathcal{E}^{[0,1] \times] 0,1]}(\bar{\Omega})$ contains the representatives of our expected approximate solutions and $\mathcal{C}$ is overgenerated by some elements connected to equation and data singularities. Moreover, these singularities also are connected to the required $\left(\Phi_{p^{-}} E\right.$ or $\Psi-E$ ) approximation processes.

\section{References}

[1] H.A. Biagioni and M. Oberguggenberger, Generalized solutions to Burgers' equation, J. Differential Equations, 97(2) (1992), 263-287, MR 93j:35151, Zbl 0777.35071.

[2] J.-J. Cauret, J.-F. Colombeau and A.Y. Le Roux, Discontinuous generalized solutions of nonlinear nonconservative hyperbolic equations, J. Math. Anal. Apl., 139 (1989), 552-573, MR 91c:35085, Zbl 0691.35057.

[3] J.-F. Colombeau, Elementary introduction to new generalized functions, NorthHolland Math. Studies, 113, 1985, MR 87f:46064, Zbl 0584.46024.

[4] V.G. Danilov and G.A. Omel'yanov, Calculation of the singularity dynamics for quadratic nonlinear hyperbolic equations. Example the Hopf equation, Nonlinear Theory of Generalized Functions, Research Notes in Mathematics Series, Chapman \& Hall/CRC, 1999, 63-74, CMP 1699 862, Zbl 0932.35144.

[5] A. Delcroix and D. Scarpalezos, Sharp topologies on $(\mathcal{C}, \mathcal{E}, \mathcal{P})$-algebras, Nonlinear Theory of Generalized Functions, Research Notes in Mathematics Series, Chapman \& Hall/CRC, 1999, 165-173, MR 2000f:46049, Zbl 0934.46049. 
[6] Topology on asymptotic algebras and applications, Monatsh. Math., 129 (2000), 1-14, MR 2001b:46065, Zbl 0952.46029.

[7] F. Demengel and D. Serre, Nonvanishing singular part of measure valued solutions for scalar hyperbolic equations, Comm. Partial Differential Equations, 16 (1991), 221-254, MR 92d:35186, Zbl 0733.35021.

[8] N. Djapic and S. Pilipovic, Approximated traveling wave solutions to generalized Hopf equation, Novi Sad J. Math., 29 (1999), 103-116, MR 2002g:35139.

[9] Y.V. Egorov, A contribution to the theory of generalized functions, Russian Math. Surveys, 45(5) (1990), 1-49, MR 92d:46097, Zbl 0754.46034.

[10] L. Hörmander, Lectures on Nonlinear Hyperbolic Differential Equations, Mathématiques \& Applications, 26, Springer-Verlag, 1997, MR 98e:35103, Zbl 0881.35001.

[11] J.-A. Marti, Fundamental structures and asymptotic microlocalization in sheaves of generalized functions, Integral Transforms Spec. Funct., 6(1-4) (1998), 223-228, MR 99f:58187, Zbl 0902.18005.

[12] _ Fixed points in algebras of generalized functions, International Conference ICIMAF, La Habana Cuba, 1997.

$[13] \_,(\mathcal{C}, \mathcal{E}, \mathcal{P})$-sheaf structures and applications, Nonlinear Theory of Generalized Functions, Research Notes in Mathematics Series, Chapman \& Hall/CRC, 1999, 175186, MR 2000f:46050, Zbl 0938.35008.

[14] J.-A. Marti and P. Nuiro, Génération d'algèbres différentielles et applications, Proceedings of the $3^{\text {rd }}$ Catalan Days on Applied Mathematics, Lleida, Espagne, 1996, 139-150, MR 99c:46037, Zbl 0897.46022.

[15] __, Analyse algébrique d'un problème de Dirichlet non-linéaire et singulier, Topol. Methods Nonlinear Anal., 13 (1999), 301-311, MR 2001e:35070, Zbl 0959.46028.

[16] J.-A. Marti, S.P. Nuiro and V.S. Valmorin, On a nonlinear Goursat problem, Integral Transforms Spec. Funct., 6(1-4) (1998), 229-246, MR 99d:35025, Zbl 0912.35043.

[17] _ Sur un probleme de Goursat non-linéaire à données irrégulières, Annales de la Faculté des Sciences de Toulouse, VII(1) (1998), 135-159, MR 99k:35012, Zbl 0915.35006.

[18] V.P. Maslov and G.A. Omel'yanov, Geometric Asymptotics for Nonlinear PDE I, Translations of Mathematical Monographs, 202, A.M.S., 2001, MR 2002k:35280, Zbl 0984.35001.

[19] M. Nedeljkov, Infinitely narrow soliton solutions to scalar conservation law in Colombeau sense, Integral Transforms and Special Functions, 6(1-4) (1998), 257-263, MR 99d:35099, Zbl 0911.35030.

[20] Ya.V. Radyno, Extensions of algebras, mnemofunctions and their applications, Nonlinear Theory of Generalized Functions, Research Notes in Mathematics Series, Chapman \& Hall/CRC, 1999, 209-218, MR 2000j:46082, Zbl 0938.46044.

Received June 30, 1999.

DÉPARTEMEnt De Mathématiques et Informatique

Université Antilles-Guyane

97157 Pointe À Pitre

GuAdELOupe

FRENCH WEST INDIES)

E-mail address: jean-andre.marti@univ-ag.fr 